Jean-Luc Gilles

Haute école pédagogique du canton de Vaud,

Lausanne

Pierre Charlier

Inspecteur général honoraire de la Ville de Liège

Sandra Pfeuti

Haute école pédagogique du canton de Vaud,

Lausanne

Oliver Prosperi

Haute école pédagogique du canton de Vaud,

Lausanne

\section{Appréciations par les élèves de leur environnement socioéducatif et résultats aux \\ épreuves externes communes en fin d'enseignement primaire : comparaison des établissements Freinet et traditionnels de l'enseignement communal liégeois}

Assessment by pupils of their socioeducational environment and results at common external tests at the end of primary education: a comparison of Freinet and traditional schools from the Liège communal public school system ésumé

La controverse sur les avantages de la pédagogie Freinet perdure depuis des décennies tant pour les valeurs ajoutées apportées en matière d'environnement socioéducatif qu'en ce qui concerne les compétences scolaires acquises. Notre étude menée dans l'enseignement communal de la ville de Liège (Belgique) où plus de 1500 élèves sont inscrits dans des établissements Freinet ( $14 \%$ de la population scolaire) montre que, à niveau socioéconomique équivalent, les élèves de $3 \mathrm{e}$ cycle des établissements Freinet obtiennent des niveaux de résultats aux épreuves communes de français, mathématiques et éveil similaires à ceux des écoles traditionnelles, mais s'en distinguent par leurs déclarations systématiquement plus positives en ce qui concerne leur environnement socioéducatif.

\section{Mots-clés}

Méthode active, réussite scolaire, climat de la classe, résultat d'évaluation, environnement socioculturel, étude comparative.

Abstract

The controversy over the advantages of Freinet pedagogy has persisted for decades in terms of both the added value of socio-educational environment and level of academic proficiency. Our study carried out in the communal public school system of the city of Liège (Belgium), where more than 1500 pupils are enrolled in Freinet schools (14\% of the school population) shows that at equivalent socio-economic level, pupils of Freinet schools obtain performance levels in French, maths and early-learning studies similar to those of traditional schools, but are distinguished by their systematically more positive statements in their socio-educational environment.

Keywords

Active learning method, academic achievement, classroom environment, assessment results, socio-cultural environment, comparative study.

\section{Introduction}

La pédagogie Freinet occupe un espace singulier dans le paysage pédagogique de nombreux pays. Née il y a une centaine d'années, elle continue d'occuper une place certes minoritaire, mais constante, voire en progression. Beaucoup de ses partisans affirment que les valeurs et les démarches pédagogiques Freinet conservent toute leur pertinence et qu'elles proposent des réponses adaptées à un projet d'école visant à favoriser l'expression libre, la créativité, l'auto-apprentissage, le dialogue et le partage de projets communs. Elles suscitent aussi l'appropriation par les élèves de méthodes de recherches personnelles et l'organisation autonome du travail. Elles proposent également une planification des apprentissages adaptée à chaque enfant et permettent la réconciliation de certains d'entre eux rebutés par les méthodes traditionnelles, tout comme elles les initient à la démarche scientifique et à l'esprit critique par le tâtonnement expérimental. Enfin, elles jettent les bases de la démocratie par la coopération, valeur-clé chez Freinet sur les plans politique et pédagogique (Acker, 2006; Freinet, 1946a, 1946b, 1969, 1978; Go, 2006; Peyronie, 2013). Pour les tenants de cette pédagogie, les écoles Freinet produisent une valeur ajoutée en termes de coopération, de solidarité, d'autonomie, de climat d'école, ou encore de compétences citoyennes difficiles à mesurer.

Mais une pédagogie alternative comme la forme scolaire «Freinet » est-elle réellement efficace? Dispose-t-on de données probantes à ce sujet? Par delà ces questions, certains interrogent ces pratiques pédagogiques en rupture avec l'école traditionnelle et postulent qu'elles pourraient générer des inégalités plutôt que de les combattre. Ils dénoncent le manque d'opportunités offertes aux élèves confrontés aux pédagogies actives d'investir cette dimension 
qui consiste à relier les gestes concrets de l'étude aux réels enjeux des apprentissages, aux objets de savoir. Il y aurait comme un défaut de secondarisation des premiers niveaux des tâches qui leur sont demandées de «faire ». Autrement dit, les pédagogies actives enfermeraient trop souvent les élèves, et en particulier les enfants de milieux populaires, dans un faire « faire » sans parvenir à les conduire vers un véritable travail d'apprenant leur permettant de prendre conscience des apprentissages qui se cachent derrière les tâches effectuées (Bautier, Charlot et Rochex, 2000; Bautier et Rochex, 1997, 2004; Bonnéry, 2007).

Goigoux (2010) relève à ce propos :

Les thèses pédagogiques inspirées par l'Éducation nouvelle [...] sont aujourd'hui contestées lorsquelles conduisent à une fréquentation trop aléatoire, trop peu hiérarchisée, trop espacée et trop peu fréquente des savoirs visés par l'enseignement. Leurs défauts de planification sont pointés du doigt, ainsi que l'habillage des situations pseudo concrètes qui conduisent les élèves à s'égarer sur de fausses pistes cognitives et à perdre les bénéfices qu'offrent une sélection, une présentation et une organisation rigoureuses de situations didactiques «artificielles » : matériel épuré, stabilité des formats des tâches scolaires, découpage des contenus, rythme de progression et gradation des difficultés, etc. (p. 23-24)

Pour certains, les établissements Freinet seraient moins efficaces que les écoles traditionnelles du point de vue des résultats scolaires. Pour ses partisans, la forme scolaire Freinet offrirait une série d'avantages au niveau de l'environnement socioéducatif par rapport à l'école traditionnelle. Mais qu'en est-il vraiment? Force est de constater que peu de recherches empiriques se sont penchées sur la question.

\section{Contexte de l'enseignement de la ville de Liège}

La création progressive, depuis 1985, d'un réseau d'écoles Freinet dans l'enseignement communal liégeois constitue un véritable laboratoire grandeur nature concernant actuellement plus de 1500 élèves, soit $14 \%$ de la population scolaire au sein de chaque niveau d'enseignement de la ville (maternel, primaire et secondaire). La mise en place progressive de ce réseau permet d'identifier notamment les conditions d'émergence, de développement et de régulation d'un dispositif pédagogique innovant dans le cadre de l'enseignement public (Charlier, 2014).

Par ailleurs, la coexistence d'écoles Freinet et d'écoles traditionnelles sur un même territoire constitue une réelle opportunité pour mener des recherches comparatives sur l'existence possible de valeurs ajoutées qui seraient propres aux écoles Freinet, en appariant celles-ci à des écoles classiques sur base de l'indice socioéconomique (ISE) calculé pour chaque établissement par la Fédération Wallonie-Bruxelles. L'indice ISE est attribué à chaque élève « sur la base des caractéristiques socioéconomiques du quartier où il vit, [...] la plus petite entité administrative pour laquelle des données sociodémographiques sont disponibles » (Fédération Wallonie-Bruxelles, 2012,p. 26). Dans un contexte belge de « quasi-marché » scolaire où les parents peuvent inscrire leurs enfants dans l'établissement de leur choix, l'appariement des écoles traditionnelles et Freinet à l'aide de cet indice ISE basé sur le quartier où les élèves vivent nous permet de répondre à certaines remarques parfaitement fondées sur des comparaisons qui pourraient être pour le moins hâtives si elles ne tenaient pas compte de cet indice ISE (Saint-Luc, 2011, p. 32). 
Cependant, même si l'indice ISE est déjà d'une très grande utilité dans cette étude comme dans d'autres, il ne permet pas d'aller jusqu'à un grossissement permettant de saisir le niveau de détails socioéconomiques liés à la famille de chaque enfant pris individuellement et reste donc une mesure perfectible.

\section{Nos questions de recherche}

Saisissant l'opportunité offerte par le contexte liégeois où, au sein du même réseau d'enseignement communal, des écoles Freinet et traditionnelles se côtoient, notre recherche vise à comparer d'une part les résultats des élèves aux épreuves de référence et d'autre part leur ressenti en ce qui concerne le climat de classe dans ces deux formes scolaires, et ce, en ciblant plus particulièrement les classes de fin du primaire.

Nous avons également saisi une autre opportunité liée à la disponibilité de l'indice socioéconomique «ISE » décrit dans le Décret édicté en 2006 organisant un encadrement différencié au sein des établissements scolaires de la Fédération Wallonie-Bruxelles afin d'assurer à chaque élève des chances égales d'émancipation sociale dans un environnement pédagogique de qualité. Cet indice permet de classer les établissements scolaires de la Fédération sur une échelle en fonction du niveau socioéconomique moyen des quartiers d'habitation des élèves. Il est calculé au moyen d'une formule :

[...] prenant en compte les dernières données statistiques disponibles pour les critères suivants : $1^{\circ}$ Revenu par habitant; $2^{\circ}$ Niveau des diplômes; $3^{\circ}$ Taux de chômage, taux d'activité et taux de bénéficiaires du revenu mensuel minimum moyen garanti; $4^{\circ}$ Activités professionnelles; $5^{\circ}$ Confort des logements (CDA, 2009, p. 2).

L'indice ISE nous offre dès lors la possibilité de comparer les résultats obtenus dans des établissements "Freinet» et «traditionnels » dont les élèves vivent dans des environnements socioéconomiques similaires.

Dans ce contexte, nous formulons nos questions de recherche de la façon suivante :

- Observe-t-on des différences de niveau des résultats aux épreuves de référence chez les élèves de fin du primaire selon qu'ils font partie d'une école Freinet ou d'une école dite traditionnelle de même catégorie ISE dans le réseau d'enseignement communal de la ville de Liège?

- $\quad$ Au sein d'une même catégorie ISE, le climat de classe diffère-t-il dans les écoles Freinet en comparaison avec les écoles traditionnelles de l'enseignement communal liégeois?

\section{Cadrage théorique}

De nombreux ouvrages consacrés à la pédagogie Freinet sont référencés, voire diffusés, à travers des associations telles que l'Institut coopératif de l'école; la Fédération internationale des mouvements d'école moderne ou encore les amis de Freinet. Dans cette profusion d'écrits, très peu de textes concernent des recherches qui ont tenté d'apporter un éclairage scientifique aux comparaisons entre écoles «Freinet» et écoles «traditionnelles ». La revue de la littérature en sciences de l'éducation 
montre également qu'il n'existe guère d'études empiriques s'intéressant à la question d'une éventuelle valeur ajoutée apportée par les écoles Freinet en matière de climat socioéducatif en comparaison avec d'autres écoles dans un contexte scolaire donné.

Nous n'avons pas trouvé d'articles référencés dans la base de données Google Scholar ni dans l'Education Resources Information Center (ERIC) concernant des recherches comparant écoles à pédagogie Freinet et écoles traditionnelles de l'enseignement primaire. Les mots-clés "Freinet » ou «Freinet pedagogy » n'existant pas dans les thesaurus, nous avons utilisé d'autres descripteurs tels que «alternative education» que nous avons croisé avec "comparative study», mais ces recherches ne donnent pas de résultats lorsqu'on cible l'enseignement primaire.

En langue française, une étude méthodologiquement très rigoureuse se démarque cependant : celle menée durant 5 ans par Reuter et son équipe dans une école de la banlieue lilloise et consignée dans l'ouvrage collectif intitulé Une école Freinet-Fonctionnements et effets d'une pédagogie alternative en milieu populaire (Reuter, 2007). En langue anglaise, nous avons aussi trouvé celle de De Bilde, Van Damme, Lamote et De Fraine (2013), une étude longitudinale portant sur 2776 élèves d'écoles traditionnelles et alternatives (Freinet et Waldorf) en Flandre (Belgique).

À propos de la littérature liée à la forme scolaire Freinet, Reuter (2007) déplore l'absence d'analyses précises conformes aux normes de scientificité décrivant les effets de la pédagogie Freinet:

Elles demeurent en effet, bien trop souvent encore, prises dans des jugements globalisants - que ce soit ceux, laudatifs, de leurs thuriféraires ou ceux, dépréciatifs, de leurs contempteurs, la plupart du temps non étayés par des investigations empiriques un tant soit peu conséquentes. (p. 9)

De leur côté, De Bilde et al. (2013) s'étonnent aussi du peu d'études, de leurs faiblesses méthodologiques et des résultats mitigés : "Surprisingly, the effects of these alternative primary schools have seldom been the subject of research, and the research that does exist has methodological limitations or has revealed mixed results » (p. 212).

\section{La question des éventuelles valeurs ajoutées de la pédagogie Freinet}

Parmi les huit travaux au centre de la recherche de l'équipe de Reuter, une étude menée par Carra et Pagoni s'intéresse plus particulièrement aux phénomènes de violence chez les élèves, à la question des normes et de la socialisation scolaires ainsi qu'au climat d'école. Elles ont eu recours, entre autres outils d'investigation, à un questionnaire administré aux élèves de l'école Freinet, à ceux d'une école témoin aux caractéristiques similaires et à 2000 autres élèves d'un échantillon représentatif. Dans les conclusions de leur article, ces chercheuses mentionnent :

La participation des élèves à la régulation de la vie scolaire, l'application des règles qu'ils votent, l'impartialité des sanctions à l'encontre de ceux qui les transgressent se traduisent par un sentiment de justice particulièrement fort dans cette école, tout en contribuant à l'adhésion des élèves à cette configuration pédagogique. (Carra et Pagoni, cité dans Reuter, 2007, p. 58) 
D'autres études du collectif de chercheurs dirigé par Reuter ont également attiré notre attention par la portée de leurs conclusions en ce qui concerne les effets de la pédagogie Freinet sur les apprentissages des élèves. Lahanier-Reuter (2007) indique :

Létude des évaluations nationales menée par R. Hassan confirme que les connaissances des élèves de Freinet en mathématiques sont comparables à celles des autres écoles du secteur, voir sur certains domaines - la géométrie les surpassent. [...] ce dispositif pédagogique particulier a des effets confirmés sur les apprentissages réalisés, sur les façons dont les enfants deviennent des élèves, sur les façons de vivre la classe. (p. 215)

En plus de la question des éventuelles valeurs ajoutées des écoles Freinet en matière de climat de classe relevées par l'équipe de Reuter, nous nous sommes également interrogés sur l'équivalence ou non des résultats aux épreuves communes en français, mathématiques et éveil des élèves fréquentant les classes Freinet comparées aux résultats des élèves des écoles traditionnelles.

Dès lors, dans une première partie de notre recherche, nous avons examiné les environnements socioéducatifs des établissements Freinet et traditionnels en questionnant les élèves. Dans l'autre partie concernant les résultats aux épreuves communes, nous comparons les résultats aux tests de référence de fin du primaire. Dans les deux parties de notre étude, les écoles comparées sont similaires du point de vue du niveau socioéconomique des quartiers d'habitation des élèves mesuré avec l'indice ISE.

\section{La conceptualisation de l'environnement socioéducatifselon Janosz, Georges et Parent (1998)}

Pour évaluer l'environnement socioéducatif des écoles de notre échantillon, nous avons utilisé le Questionnaire sur l'environnement socioéducatif(QES) du Groupe de recherche sur les environnements scolaires de l'Université de Montréal. Cet instrument issu d'un programme de recherche et développement débuté au milieu des années 1990 propose une série d'items permettant de tracer le portrait d'une école en ce qui concerne son climat, ses problèmes et ses pratiques éducatives.

Concernant les raisons liées au choix de l'outil QES plutôt qu'un autre dans le cadre de cette recherche, relevons d'abord qu'il s'agit d'un instrument élaboré à partir d'une conceptualisation solide et dont la validité et les qualités édumétriques ${ }^{1}$ sont solidement établies (Janosz, 2000; Janosz, Bouthillier, Bowen, Chouinard et Desbiens, 2007; Janosz et al., 1998). À notre connaissance, il n'existe pas d'autre questionnaire en langue française qui incorpore les différentes dimensions que nous souhaitions investiguer : climat scolaire, pratiques éducatives de l'école et problématiques perçues et vécues dans l'école. Enfin, c'est un questionnaire qui existe dans une version pour l'enseignement secondaire, mais aussi dans une version pour l'école primaire.

Notons qu'il existe un autre instrument, plus ancien : le Classroom Environment Scale (CSE) de Moos et Trickett (1974) dont une traduction existe en québécois. Cependant, comme le signalent Janosz et al. (1998) : 
Le CSE constitue un instrument approprié quand il s'agit d'évaluer le contexte scolaire d'un élève en particulier, d'évaluer le fonctionnement d'une classe spécifique, ou pour déterminer si les problèmes des jeunes se limitent à certaines classes, mais il accuse d'importantes lacunes si la centration porte davantage sur l'école. (p. 288)

Or, notre recherche se situe dans une perspective comparatiste d'environnements socioéducatifs d'écoles Freinet et traditionnelles. Il existe aussi un instrument d'évaluation du climat social d'une école, le Effective School Battery (ESB) (Gottfredson, 1984), qui offre la possibilité d'établir le profil particulier d'un établissement scolaire. Cet instrument validé est très utilisé aux États-Unis, mais il n'est pas traduit en français.

Concernant le QES et l'évaluation du climat scolaire dans l'environnement socioéducatif, l'équipe de Janosz distingue cinq composantes: le climat relationnel, l'atmosphère qui règne entre les individus; le climat éducatif, la valeur accordée à l'éducation dans le milieu; le climat de sécurité, l'ordre et la tranquillité du milieu; le climat de justice, juste appréciation des droits et devoirs de chacun et le climat d'appartenance.

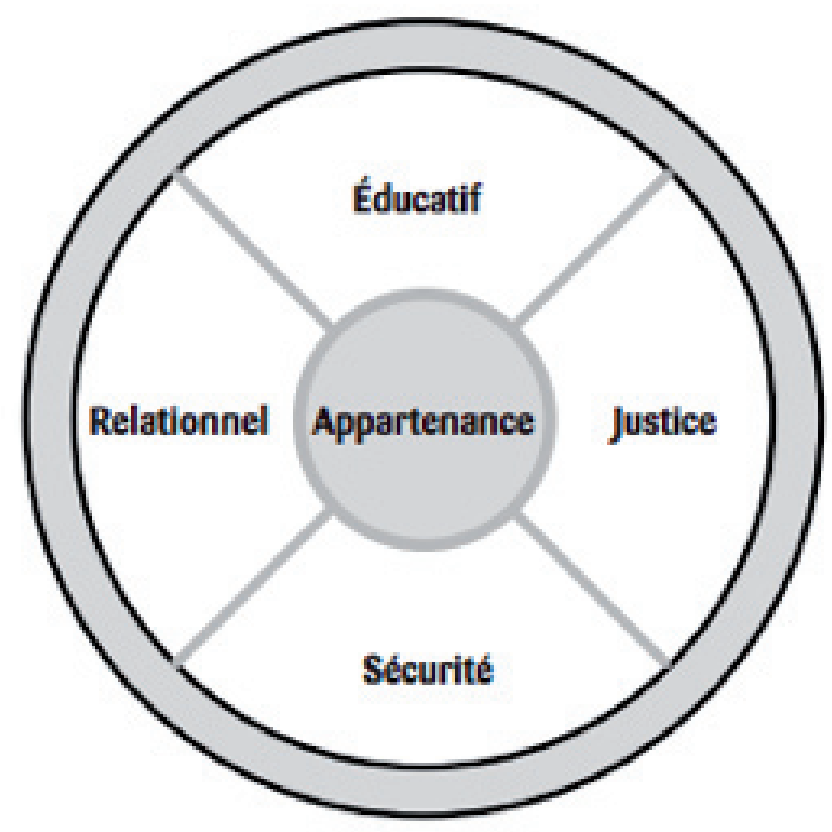

Figure 1

Composante de l'environnement socioéducatif : le climat scolaire et ses différentes facettes selon Janosz et al. (1998, p. 293) 
Concernant le climat d'appartenance, les auteurs précisent que cette dimension centrale transcende les autres composantes :

[...] en ce qu'elle se développe à partir des autres types de climats. Lorsque les individus ont l'impression que leur milieu est porteur de sens, qu'il favorise le contact humain, qu'il assure leur protection et qu'il garantit la reconnaissance de leurs droits et de leurs efforts au même titre qu'il sanctionne de façon juste et équitable leurs transgressions à la norme, il développe un sentiment d'appartenance. (Janosz et al., 1998, p. 294)

Concernant les pratiques éducatives de l'école, Janosz et al. (1998) ont retenu huit catégories de pratiques mises en avant par les résultats de recherches empiriques:

[...] l'encadrement des élèves; l'emphase sur la réussite éducative des élèves; le système de reconnaissance; la qualité et le temps consacré à l'enseignement, les occasions d'investissement scolaire et parascolaire, la participation des parents et le leadership éducatif de même que le style de gestion de la direction. (p. 295)

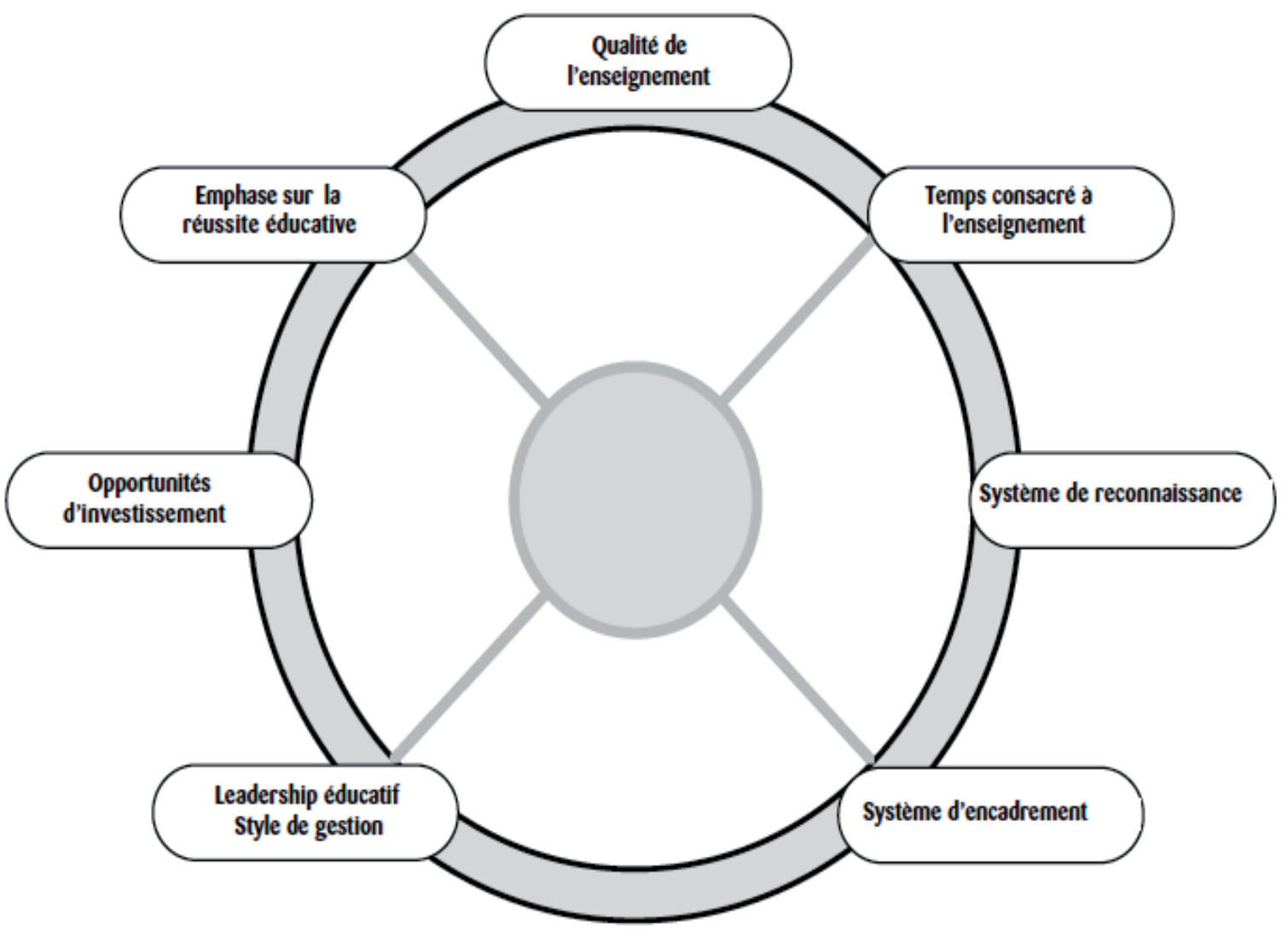

Figure 2

Composante de l'environnement socioéducatif: les pratiques éducatives selon Janosz et al. (1998, p. 295) 
Enfin, Janosz et al. (1998) proposent de distinguer les problèmes scolaires selon qu'ils relèvent d'une part de la sphère scolaire en distinguant les problèmes des élèves de ceux des enseignants ou d'autre part d'une socialisation plus générale.

Dans le cadre de notre recherche, nous avons repris le Questionnaire sur l'environnement socioéducatif (QES) de Janosz, Bowen, Chouinard et Desbiens (2004) conçu à partir de cette conceptualisation pour le Québec. Nous l'avons légèrement adapté du point de vue du vocabulaire au contexte francophone belge à partir de la troisième version testée par l'équipe québécoise et destinée aux écoles primaires.

Instruments de collecte des données

Le premier volet de notre enquête consiste en la collecte de données auprès des élèves à l'aide d'un questionnaire auto-administré relatif à l'environnement scolaire et ses dimensions sociale et éducative. Le second volet est quant à lui constitué par les résultats aux épreuves externes communes 2009 à 2011.

\section{Le questionnaire sur l'environnement socioéducatif}

Le Questionnaire sur l'environnement socioéducatif pour le niveau primaire que nous avons utilisé est le fruit d'années de recherches menées par le professeur Michel Janosz et ses collaborateurs de l'Université de Montréal. Le QES-primaire est issu du QES-secondaire qui est lui-même issu d'un instrument mis au point en 1998 et intitulé «Le climat d'école» (Janosz et al., 1998). Janosz et son équipe ont non seulement effectué une recherche théorique, notamment via une large revue de la littérature, mais également mené une série d'études empiriques qui ont permis de déterminer les propriétés psychométriques des échelles de mesure qui visent à rendre compte du construit théorique. Dans leur rapport de validation du QES-primaire (Janosz et al., 2007), les auteurs décrivent en détail le contexte théorique sous-jacent ainsi que le développement de l'outil et les opérations de validation (Janosz et al., 2007, p. 2-36).

C'est la dernière version du questionnaire issue de la phase trois que nous avons utilisée : «troisième version de l'instrument qui a atteint les standards de qualité que nous nous étions fixés " (Janosz et al., 2007, p. 5). Plusieurs facettes du climat scolaire sont abordées dans le questionnaire : le climat relationnel (entre les élèves, entre l'élève et l'enseignant), le climat de justice (équité perçue et façon dont les élèves se sentent traités à l'école), le climat d'appartenance (fierté à fréquenter l'établissement et son importance en tant que lieu de vie) et le climat de sécurité chez les élèves (perception sur la sûreté des élèves, l'ordre et la tranquillité du milieu ainsi que les risques perçus de victimisation).

Les pratiques éducatives de l'école évaluées par le QES couvrent le système d'encadrement de l'école (implantation et clarté des règles), les pratiques pédagogiques, la gestion des comportements en classe (y compris le temps consacré à l'enseignement et la motivation des enseignants), l'aide offerte aux élèves éprouvant des difficultés scolaires ou personnelles, la participation des élèves à la vie de l'école ainsi que la collaboration entre l'école et la famille. Le QES évalue aussi la fréquence perçue des problèmes à l'école concernant des comportements d'inadaptation scolaire et des problèmes de violence perçus, voire subis, de gravité mineure ou majeure. 
La version utilisée dans cette recherche comporte au total 123 questions présentées en huit grandes parties, déclinées en deux à cinq thématiques. En comparaison avec la version originale, nous avons supprimé 25 questions, parce qu'elles ne présentaient pas d'intérêt ou n'étaient pas utiles, par exemple l'item sur la fréquence d'utilisation de l'autobus scolaire qui n'est pas un service offert aux élèves dans l'enseignement communal liégeois.

\section{Épreuve externe des évaluations des acquis}

Le second volet de l'enquête est constitué par les résultats aux épreuves externes communes de 2009 à 2011. Ces évaluations certificatives des acquis des élèves, organisées par la Fédération WallonieBruxelles, sont obligatoires pour tous les élèves de tous les réseaux. Elles concernent tous les élèves de sixième année primaire et sont liées à l'octroi du certificat d'études de base (CEB).

Quatre disciplines réparties en onze domaines sont évaluées :

- $\quad$ le français (Lecture, Outils au service de la langue², Savoir écouter et Savoir écrire);

- $\quad$ la formation mathématique (Nombres et opérations, Solides et figures, Grandeurs et Traitement de données);

- l'éveil-initiation scientifique/Sciences;

- léveil-formation historique et géographique/Histoire-géographie.

Les scores sont calculés en points et transformés en pourcentages puis communiqués discipline par discipline.Pour plus de détails sur les épreuves du CEB, nous renvoyons le lecteur au site « enseignement. be » de la Fédération Wallonie-Bruxelles qui présente les questionnaires, le portfolio, les consignes de passation et de correction ainsi que le dossier de présentation pour toutes les épreuves depuis $2007^{3}$

\section{Échantillonnage des écoles}

Parmi la quarantaine d'établissements scolaires publics de la ville de Liège, cinq sont de type Freinet ${ }^{4}$. Toutes les écoles Freinet en place en 2013 au sein de l'enseignement communal liégeois sont comprises dans l'échantillon. Pour le choix des écoles traditionnelles, nous avons opéré une sélection en fonction des possibilités d'appariement avec chaque école Freinet, aboutissant ainsi à un échantillon total de dix écoles. Cet appariement a été fait sur la base de l'indice socioéconomique ISE en associant à chaque école Freinet un établissement traditionnel choisi au hasard parmi ceux ayant un indice proche de celui de l'école Freinet considérée. Le choix des écoles traditionnelles a donc été opéré en fonction de leur niveau ISE correspondant au niveau ISE des écoles Freinet. Aucune école n'a refusé de participer à cette étude. Le questionnaire a été soumis à toutes les classes de $5^{\mathrm{e}}$ et de $6^{\mathrm{e}}$ des établissements de l'échantillon. Les données relatives aux enseignants des classes sélectionnées ne sont pas fournies pour des raisons liées à l'anonymisation. Le tableau suivant montre la répartition des sujets en fonction du type d'établissement et du niveau de scolarité. 


\section{Tableau 1}

Nombre de sujets par type d'établissement et par niveau scolaire

\begin{tabular}{l|c|c|c}
\hline Forme d'enseignement & \multicolumn{1}{c}{$\mathbf{5}^{\mathbf{e}}$ année primaire } & \multicolumn{1}{c}{ Totaux } \\
\hline Freinet & $\mathrm{N}=101$ & $\mathrm{~N}=95$ & $\mathrm{~N}=196$ \\
Traditionnel & $\mathrm{N}=101$ & $\mathrm{~N}=93$ & $\mathrm{~N}=194$ \\
Totaux & $\mathrm{N}=202$ & $\mathrm{~N}=188$ & $\mathrm{~N}=390$ \\
\hline
\end{tabular}

\section{Collecte des données}

\section{Recueil de données dans le cadre du QES}

Les élèves ont répondu au questionnaire sur une base strictement anonyme. La passation s'est déroulée en classe, les 24 et 25 juin 2013, et toutes les questions ont été lues aux élèves par une personne autre que le titulaire et en dehors de sa présence. En raison de la longueur du questionnaire, la passation s'est déroulée sur deux périodes de 50 minutes, entrecoupées d'une pause d'une vingtaine de minutes (récréations).

Un protocole de recueil des données a été transmis et expliqué lors d'une rencontre de préparation avec les 13 personnes qui ont assuré la passation du questionnaire.

Les directions d'établissement, les titulaires de classe et les parents d'élèves ont également été contactés et renseignés sur la démarche par l'inspection pédagogique de l'enseignement communal liégeois.

Procédure de recueil des données dans le cadre de l'épreuve externe commune

Les épreuves externes communes sont encadrées par un protocole strict en Fédération WallonieBruxelles (FWB). Lors de ces épreuves, les inspecteurs de la FWB distribuent les livrets sous boîtes scellées aux directions de l'école et organisent les corrections. Celles-ci sont réalisées à l'aide d'une grille détaillée par un groupe composé des enseignants (les titulaires ne corrigent pas les épreuves de leurs élèves) et des directions volontaires. Les épreuves 2009-2011 se sont déroulées dans la seconde partie du mois de juin.

\section{Analyse des données du QES}

Nous présentons dans cette section nos analyses des données récoltées. Le tableau ci-dessous reprend les dimensions, variables et nombre d'items du questionnaire $\mathrm{QE}^{5}$. 


\section{Tableau 2}

Dimensions et variables composant les échelles du Questionnaire sur l'environnement socioéducatif (QES) (Janosz et al., 2004)

\begin{tabular}{|c|c|c|}
\hline Dimensions de l'environnement socioéducatif et leurs variables & Acronymes & $\begin{array}{l}\text { Nbre } \\
\text { items }\end{array}$ \\
\hline \multicolumn{3}{|l|}{ I. Climat scolaire } \\
\hline \multicolumn{3}{|l|}{ 1. Climat relationnel } \\
\hline - Relations entre les élèves & crele & 4 \\
\hline - Relations entre les élèves et les enseignants & crela & 5 \\
\hline 2. Climat de justice & cjust & 4 \\
\hline 3. Climat d'appartenance & capp & 4 \\
\hline 4. Climat de sécurité chez les élèves & $\operatorname{csec}$ & 4 \\
\hline \multicolumn{3}{|l|}{ II. Pratiques éducatives de l'école } \\
\hline 1. Implantation et clarté des règles & regi & 6 \\
\hline 2. Pratiques pédagogiques & qual & 12 \\
\hline 3. Temps consacréà l'enseignement & temp & 3 \\
\hline $\begin{array}{l}\text { 4. Gestion des comportements en classe (y compris la motivation des } \\
\text { enseignants) }\end{array}$ & pgesc & 5 \\
\hline 5. Soutien aux élèves en difficulté & sout & 4 \\
\hline 6. Participation des élèves à la vie de l'école & peve & 3 \\
\hline 7. Collaboration entre lécole et la famille & pare & 5 \\
\hline \multicolumn{3}{|l|}{ III. Problématiques perçues et vécues dans l'école } \\
\hline \multicolumn{3}{|l|}{ 1. Problèmes scolaires perçus et agis } \\
\hline - Comportements d'inadaptation scolaire perçus & prscp & 6 \\
\hline - Comportements d'inadaptation scolaire rapportés par les élèves & prsca & 6 \\
\hline \multicolumn{3}{|l|}{ 2. Problèmes de violence perçus et subis } \\
\hline \multicolumn{3}{|l|}{ - Les problèmes de violence perçus } \\
\hline . énoncés, gravité mineure & probmip & 6 \\
\hline . énoncés, gravité majeure & prmap & 7 \\
\hline $\begin{array}{l}\text { - Perception des caractéristiques des individus qui occasionnent des } \\
\text { problèmes }\end{array}$ & prcap & 2 \\
\hline \multicolumn{3}{|l|}{ - Les problèmes de violence subis } \\
\hline . énoncés, gravité mineure & prmis & 4 \\
\hline . énoncés, gravité majeure & prmas & 8 \\
\hline . rapport des problèmes de violence subis & prap & 3 \\
\hline - Moment où il y a le plus de risque d'agression & pmom & 1 \\
\hline 3. Sécurité des lieux de l'école & secl & 12 \\
\hline 4. Accessibilité aux drogues & droga & 1 \\
\hline \multicolumn{3}{|l|}{ Données supplémentaires } \\
\hline Informations générales & & 3 \\
\hline Motivation scolaire auto-révélée & vis & 5 \\
\hline
\end{tabular}




\section{Observation des moyennes}

Dans le tableau qui suit, une moyenne proche de 1 caractérise une situation plus favorable, sauf pour les variables $c s e c$, pgesc et temp où une moyenne proche de 4 indique de meilleures appréciations. Les résultats les plus élevés sont surlignés en gris. Une première observation des moyennes obtenues par forme d'enseignement pour les vingt variables des trois dimensions révèle pour la forme « école Freinet » une situation déclarée systématiquement plus favorable que pour la forme «école traditionnelle ».

\section{Tableau 3}

Moyennes, effectifs et écarts-types des 20 variables aux 3 dimensions du QES

(en gris les plus favorables)

\begin{tabular}{|c|c|c|c|c|c|c|c|c|c|}
\hline \multicolumn{2}{|c|}{ Variables de la dimension I } & \multirow{2}{*}{$\begin{array}{l}\text { crele } \\
2,19\end{array}$} & \multirow{2}{*}{$\begin{array}{l}\text { crela } \\
1,85\end{array}$} & \multirow{2}{*}{$\begin{array}{l}\text { cjust } \\
1,85\end{array}$} & \multirow{2}{*}{$\begin{array}{l}\text { capp } \\
1,76\end{array}$} & \multirow{2}{*}{$\begin{array}{l}\text { csec } \\
2,53\end{array}$} & & & \\
\hline Freinet & Moyenne & & & & & & & & \\
\hline & $\mathrm{N}$ & 195 & 192 & 195 & 193 & 193 & & & \\
\hline & Écart-type & 0,51 & 0,33 & 0,65 & 0,69 & 0,73 & & & \\
\hline \multirow[t]{3}{*}{ Traditionnelle } & Moyenne & 2,40 & 2,10 & 2,22 & 2,24 & 2,35 & & & \\
\hline & $\mathrm{N}$ & 194 & 191 & 189 & 193 & 191 & & & \\
\hline & Écart-type & 0,62 & 0,46 & 0,79 & 0,91 & 0,72 & & & \\
\hline \multirow[t]{3}{*}{ Total } & Moyenne & 2,29 & 1,97 & 2,03 & 2,00 & 2,44 & & & \\
\hline & N & 389 & 383 & 384 & 386 & 384 & & & \\
\hline & Écart-type & 0,58 & 0,42 & 0,75 & 0,84 & 0,73 & & & \\
\hline \multicolumn{2}{|c|}{ Variables de la dimension II } & regi & qual & temp & pgesc & sout & peve & pare & \\
\hline \multirow[t]{3}{*}{ Freinet } & Moyenne & 1,57 & 1,41 & 1,81 & 3,30 & 1,44 & 1,77 & 1,75 & \\
\hline & $\mathrm{N}$ & 193 & 194 & 196 & 189 & 194 & 195 & 192 & \\
\hline & Écart-type & 0,51 & 0,39 & 0,72 & 0,72 & 0,44 & 0,78 & 0,56 & \\
\hline \multirow[t]{3}{*}{ Traditionnelle } & Moyenne & 1,74 & 1,78 & 1,56 & 2,85 & 1,77 & 2,85 & 2,14 & \\
\hline & $\mathrm{N}$ & 192 & 190 & 192 & 193 & 191 & 194 & 191 & \\
\hline & Écart-type & 0,66 & 0,62 & 0,56 & 0,79 & 0,70 & 0,88 & 0,59 & \\
\hline \multirow[t]{3}{*}{ Total } & Moyenne & 1,66 & 1,59 & 1,68 & 3,08 & 1,61 & 2,31 & 1,95 & \\
\hline & $N$ & 385 & 384 & 388 & 382 & 385 & 389 & 383 & \\
\hline & Écart-type & 0,59 & 0,55 & 0,66 & 0,79 & 0,61 & 0,99 & 0,61 & \\
\hline \multicolumn{2}{|c|}{ Variables de la dimension III } & prscp & prsca & probmip & prmap & prmis & prmas & secl & vis \\
\hline \multirow[t]{3}{*}{ Freinet } & Moyenne & 1,94 & 1,25 & 2,04 & 1,46 & 1,47 & 1,26 & 1,72 & 1,33 \\
\hline & $\mathrm{N}$ & 195 & 195 & 195 & 193 & 196 & 194 & 185 & 195 \\
\hline & Écart-type & 0,57 & 0,39 & 0,57 & 0,38 & 0,51 & 0,39 & 0,61 & 0,36 \\
\hline \multirow[t]{3}{*}{ Traditionnelle } & Moyenne & 2,30 & 1,37 & 2,14 & 1,68 & 1,69 & 1,36 & 2,13 & 1,43 \\
\hline & N & 191 & 194 & 192 & 190 & 194 & 189 & 191 & 194 \\
\hline & Écart-type & 0,64 & 0,49 & 0,54 & 0,45 & 0,65 & 0,42 & 0,70 & 0,42 \\
\hline \multirow[t]{3}{*}{ Total } & Moyenne & 2,12 & 1,31 & 2,09 & 1,57 & 1,58 & 1,31 & 1,93 & 1,38 \\
\hline & N & 386 & 389 & 387 & 383 & 390 & 383 & 376 & 389 \\
\hline & Écart-type & 0,63 & 0,44 & 0,56 & 0,43 & 0,59 & 0,41 & 0,69 & 0,40 \\
\hline
\end{tabular}




\section{Tests d'hypothèse}

Les données sous-jacentes aux dimensions ne sont pas distribuées normalement et ne varient pas de la même façon. Les tests de Shapiro-Wilk pour toutes les variables et pour chaque type de forme d'enseignement («Freinet» ou «Traditionnelle») indiquent que nous ne sommes pas en présence de distributions normales. Il en est de même pour le test de Levene : nous nobservons pas d'égalité de variance, il n'est donc pas possible d'effectuer des analyses de variance classiques dans le contexte de notre étude comparative.

Nous nous sommes dès lors orientés vers des tests non paramétriques, mais avec lesquels on perd la notion de "variation d'une variable sous contrôle d'une autre variable ». Nous avons effectué des tests U de Mann-Whitney pour échantillons indépendants.

Les différences entre les distributions des vingt variables pour les deux formes d'enseignement, «école Freinet»vs "école traditionnelle», sont toutes significatives à l'exception de probmip ${ }^{6}$. Le tableau 4 expose les résultats des tests pour les vingt variables des trois dimensions de l'environnement socioéducatif comparées.

\section{Tableau 4}

Résultats des tests d'bypothèse

\begin{tabular}{ccccccccccc}
\hline & crele & crela & cjust & csec & capp & probmip & prmap & prscp & regi & peve \\
\hline $\begin{array}{c}\text { U de Mann- } \\
\begin{array}{c}\text { Whitney } \\
\text { Sig. }\end{array}\end{array}$ & 15216 & 12049 & 13171,5 & 15604,5 & 12852,5 & 16649 & 12882 & 12530,5 & 16097,5 & 7094,5 \\
\hline & 0,001 & 0,000 & 0,000 & 0,009 & 0,000 & 0,059 & 0,000 & 0,000 & 0,025 & 0,000 \\
\hline & sout & pare & temp & qual & pgesc & prmis & prmas & prsca & secl & vis \\
\hline $\begin{array}{c}\text { U de Mann- } \\
\text { Whitney } \\
\text { Sig. }\end{array}$ & 13518,5 & 11141 & 15243,5 & 11604,5 & 11979,5 & 15390,5 & 15198,5 & 15352,5 & 11475 & 16167,5 \\
\hline
\end{tabular}

\section{Taille de l'effet}

En vue de mesurer à quel point les distributions des réponses ne sont pas superposées pour les différentes variables des trois dimensions observées lorsqu'on compare l'enseignement Freinet et les écoles traditionnelles, nous avons pris en compte la taille de l'effet à l'aide du delta de Cliff.

Nous reportons dans les tableaux 5 à 7 le delta de Cliff en valeur absolue. Cet indice varie de 0 à 1 et une valeur de 0 correspond à des distributions superposées. 


\section{Tableau 5}

Taille de l'effet des différences entre écoles Freinet et traditionnelles pour les variables de la dimension "I. Climat scolaire"

\begin{tabular}{ccccc}
\hline \multicolumn{5}{l}{ Valeurs obtenues au delta de Cliff pour les dimensions de la catégorie « I. Climat scolaire» } \\
\hline crele & crela & cjust & csec & capp \\
0,20 & 0,34 & 0,29 & 0,15 & 0,31 \\
\hline
\end{tabular}

\section{Tableau 6}

Taille de l'effet des différences entre écoles Freinet et traditionnelles pour les variables de la dimension "II. Pratiques éducatives de l'école»

\begin{tabular}{ccccccc}
\hline \multicolumn{6}{c}{ Valeurs obtenues au delta de Cliff pour les dimensions de la catégorie « II. Pratiques éducatives de lécole » } \\
\hline regi & qual & temp & pgesc & sout & peve & pare \\
0,13 & 0,37 & 0,19 & 0,34 & 0,27 & 0,62 & 0,39 \\
\hline
\end{tabular}

\section{Tableau 7}

Taille de l'effet des différences entre écoles Freinet et traditionnelles pour les variables de la dimension "III. Problématiques perçues et vécues dans l'école»

Valeurs obtenues au Delta de Cliff pour les dimensions de la catégorie « III. Problématiques perçues et
vécues dans l'école »
\begin{tabular}{cccccccc} 
prscp & prsca & probmip & prmap & prmis & prmas & secl & vis \\
0,33 & 0,19 & 0,11 & 0,30 & 0,19 & 0,17 & 0,35 & 0,15 \\
\hline
\end{tabular}

Parmi les différences observées en faveur de la forme d'enseignement Freinet, la taille de l'effet la plus forte est observée pour la variable peve (Participation des élèves à la vie de l'école). Les variables crela, cjust, capp, pare, qual,pgesc, prscp, prmap et secl montrent des tailles d'effet assez marquées. En ce qui concerne crele, csec, regi, temp, sout, prsca, prmis, prmas et vis on observe aussi un effet, mais moins marqué.

\section{La participation des élèves à la vie de l'école, une variable de la dimension «Pratiques éducatives » particulièrement discriminante}

La figure suivante montre la différence des distributions des réponses des élèves pour peve selon la forme d'enseignement. Elle illustre l'influence de la forme d'enseignement sur la participation déclarée des élèves à la vie de l'école et la valeur élevée de la taille de l'effet obtenue pour cette dimension de la catégorie «II. Pratiques éducatives de l'école». Les deux distributions montrent une nette différence concernant la participation à la vie de l'école. En effet, avec un choix majoritaire de «1» pour les 3 items qui composent la dimension perve, les élèves des écoles Freinet comparés à ceux des écoles traditionnelles affirment avec plus de force leur participation à la vie de l'école. 


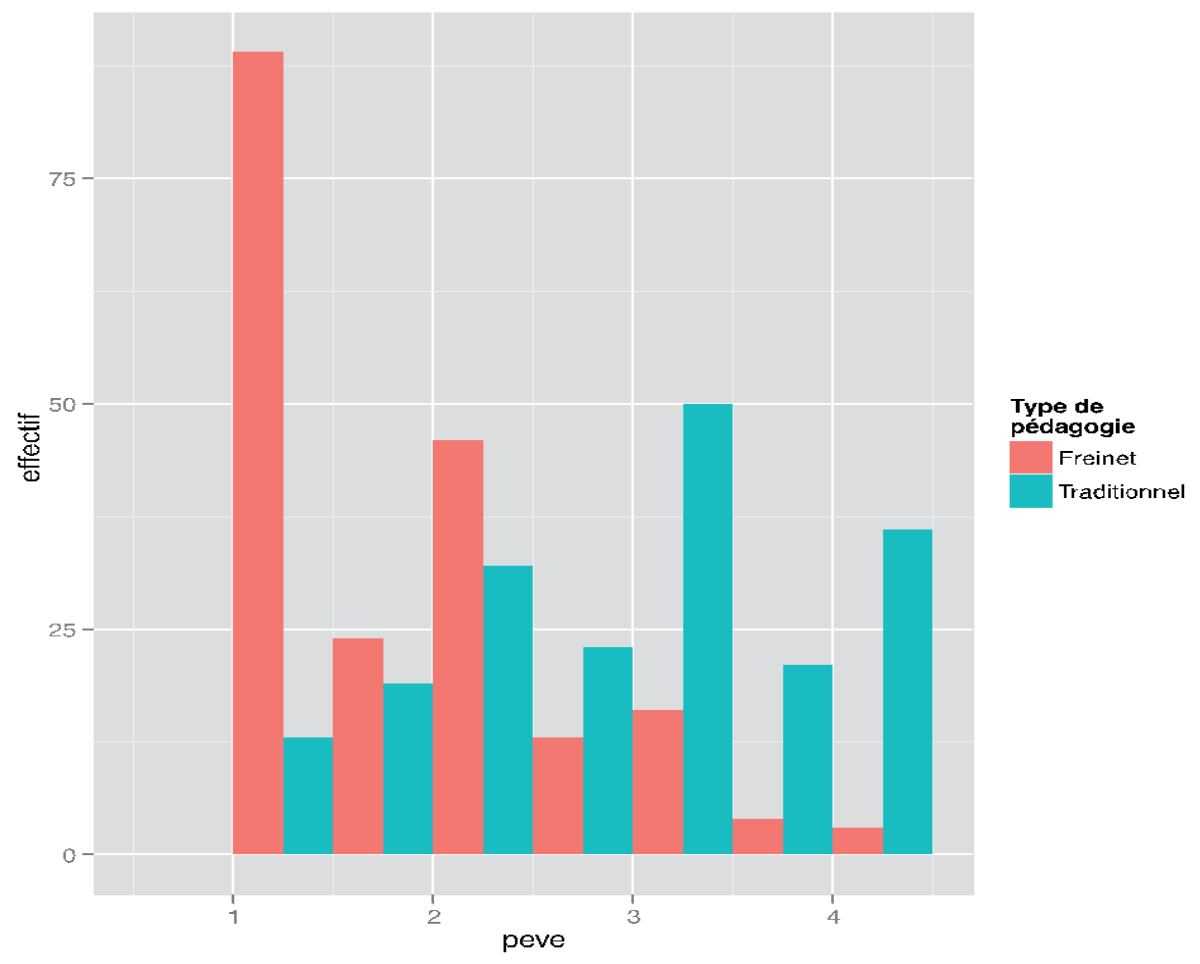

Figure 3

Distribution des réponses aux items de la variable peve « Participation des élèves à la vie de l'école » en fonction du type d'établissement « école Freinet » vs « école traditionnelle »

\section{Analyse des trois dimensions principales}

Trois dimensions principales composent le QES (tableau 2) : «I. Climat scolaire » (cscol), « II. Pratiques éducatives de l'école » (prated) et "III. Problématiques perçues et vécues dans l'école » (prob). Nous avons calculé trois variables agrégées (cscol, prated et prob) qui donnent une vision d'ensemble pour chacune de ces trois dimensions. Là où c'était nécessaire, nous avons inversé les échelles des items afin que les valeurs proches de 1 illustrent systématiquement des situations plus favorables.

L'agrégation des résultats selon ces trois dimensions permet d'observer des différences plus accentuées entre les deux formes d'enseignement.

Comme illustré dans le tableau 8 ci-dessous, les tests statistiques montrent des résultats significatifs. Le tableau 9 montre quant à lui des tailles d'effet moyennes.

\section{Tableau 8}

Tests statistiques des variables agrégées selon les trois dimensions du QES

\begin{tabular}{lccc}
\hline & cscol & prated & prob \\
\hline U de Mann-Whitney & 9759 & 7643 & 11946 \\
Sig. & 0,000 & 0,000 & 0,000 \\
\hline
\end{tabular}




\section{Tableau 9}

Tailles d'effets des variables agrégées selon les trois dimensions du QES

\begin{tabular}{ccc}
\hline \multicolumn{3}{c}{ Delta de Cliff des dimensions principales } \\
\hline cscol & prated & prob \\
0,33 & 0,39 & 0,24 \\
\hline
\end{tabular}

Les représentations graphiques des distributions des réponses aux items liés aux trois dimensions illustrent ces différences entre les deux formes scolaires, à gauche les distributions liées aux réponses des élèves dans les écoles Freinet et à droite celles qui concernent les réponses des élèves dans les écoles traditionnelles de l'enseignement communal liégeois (des valeurs proches de 1 représentent une situation plus favorable). Concernant les distributions liées au climat scolaire (cscol) on observe une répartition plus «normale » de la distribution «Freinet » avec une asymétrie vers le « 1 » tandis que la distribution de «Traditionnel» montre un pic aux valeurs 2,1 et 2,2. Pour ce qui est des distributions liées aux pratiques éducatives (prated), on observe que les deux distributions présentent des pics, mais celui de la distribution Freinet est plus proche de « 1 ». Finalement, les distributions liées aux problèmes à l'école (prob) ont une forme similaire, mais la distribution « Freinet » montre une asymétrie plus forte vers des valeurs proches de « $1 »$.

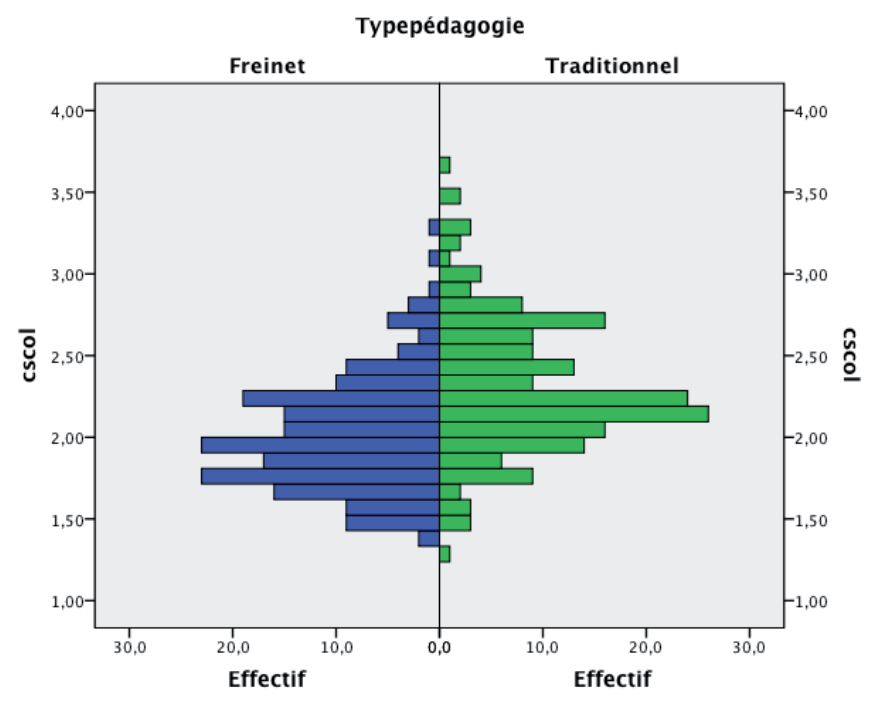

Figure 4

Distribution des réponses aux items liés à la dimension «Climat scolaire » en fonction de la forme scolaire : «Freinet » vs «Traditionnelle » 


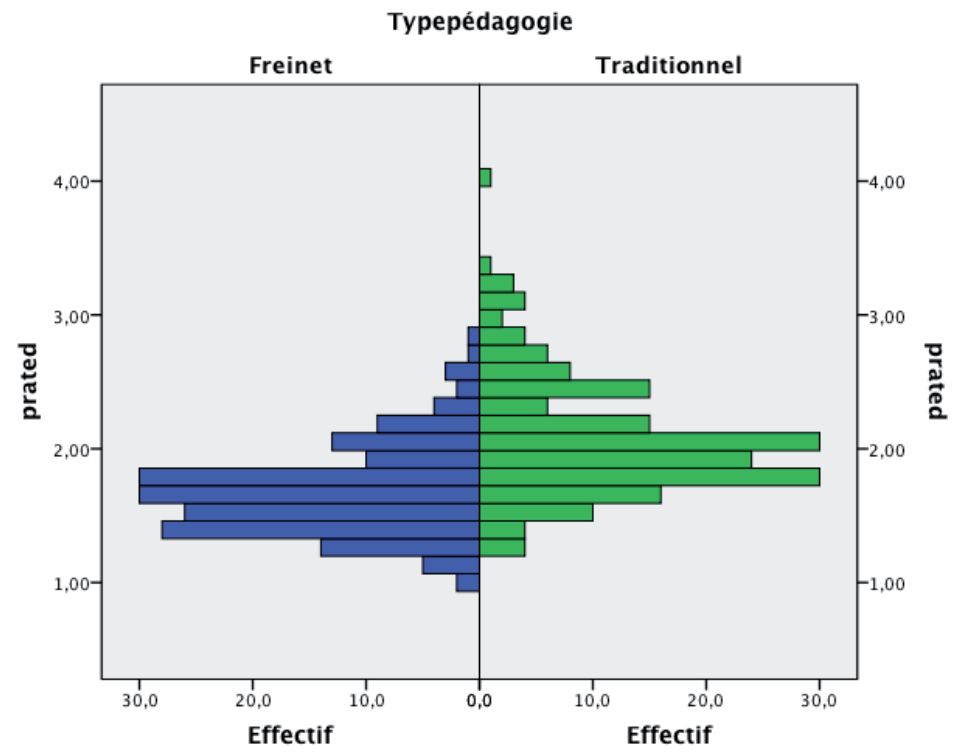

Figure 5

Distribution des réponses aux items liés à la dimension «Pratiques éducatives à l'école » en fonction de la forme scolaire : « Freinet » vs «Traditionnelle »

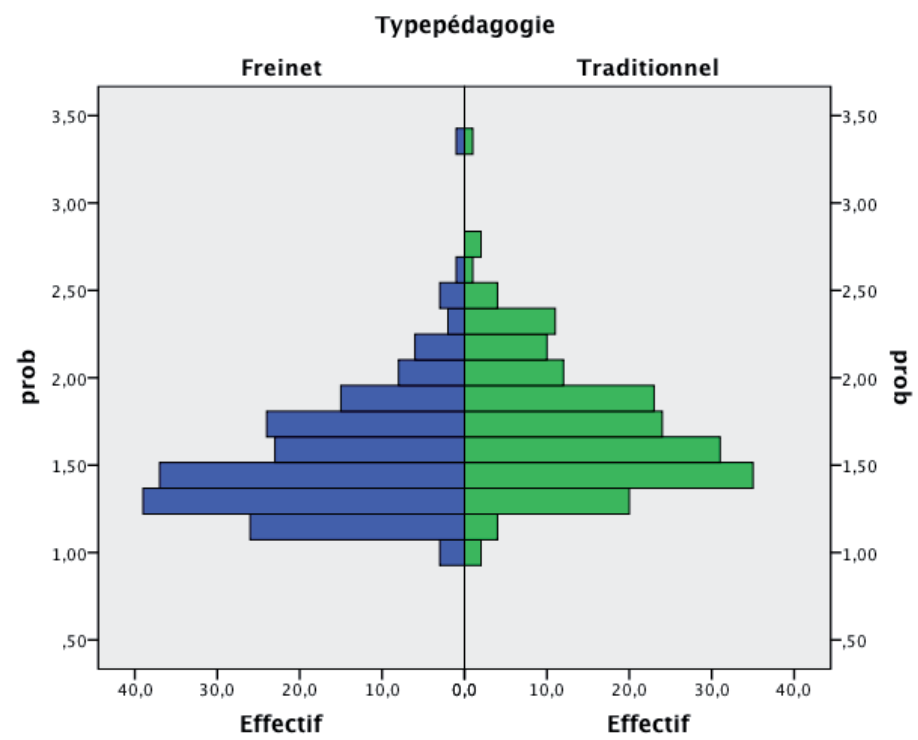

Figure 6

Distribution des réponses aux items liés à la dimension "Problématiques perçues et vécues dans l'école » en fonction de la forme scolaire : «Freinet » vs «Traditionnelle » 


\section{Résultats des épreuves communes}

Ne possédant pas les données individuelles des résultats des épreuves communes, mais seulement les moyennes par établissement et le nombre d'élèves pour les années de 2009 à 2011, nous avons procédé à une comparaison des scores moyens sur trois ans des écoles Freinet et traditionnelles ayant un indice ISE (indice socioéconomique) proche.

Les classes de chaque forme scolaire ont été réparties en deux groupes : (1) celles des établissements ayant un indice ISE de 1 ou 2 et (2) celles des établissements ayant un indice ISE de 6 ou 7. Nous avons ensuite comparé les moyennes des résultats obtenus en français, mathématiques et éveil par les élèves dans chaque type d'établissement (« Freinet » vs «Traditionnel»). Le tableau 10 résume les résultats moyens pondérés par le nombre d'élèves calculés à partir des scores obtenus de 2009 à 2011 pour ces deux groupes ISE d'établissements scolaires. Dans le contexte de cette étude où les données du CEB nous ont été livrées de façon déjà résumée, nous avons jugé plus pertinent de garder l'information sous forme de moyennes, notamment afin de garder la comparaison avec les moyennes de la Communauté française de Belgique.

\section{Tableau 10}

Comparaison des moyennespondérées des résultats des élèves desécoles Freinet et traditionnelles de l'enseignement communal liégeois aux épreuves communes 2009 à 2011 avec les moyennes de la Communauté française de Belgique et en tenant compte de l'indice socioéconomique (ISE)

\begin{tabular}{|c|c|c|c|}
\hline & Français & Mathématiques & Éveil \\
\hline \multirow[b]{2}{*}{ Groupe ISE $=1$ ou 2} & $\begin{array}{c}65,94 \\
(s d=1,4)\end{array}$ & $\begin{array}{c}66,13 \\
(s d=2,81)\end{array}$ & $\begin{array}{c}67,83 \\
(s d=5,06)\end{array}$ \\
\hline & $\begin{array}{c}66,72 \\
(s d=2,36)\end{array}$ & $\begin{array}{c}66,42 \\
(s d=1,9)\end{array}$ & $\begin{array}{c}69,72 \\
(s d=2,58)\end{array}$ \\
\hline \multirow[b]{2}{*}{ Groupe ISE = 6 ou 7} & $\begin{array}{c}76,80 \\
(s d=3,14)\end{array}$ & $\begin{array}{c}74,74 \\
(s d=1,68)\end{array}$ & $\begin{array}{c}80,47 \\
(s d=2,79)\end{array}$ \\
\hline & $\begin{array}{c}76,87 \\
(s d=2,58)\end{array}$ & $\begin{array}{c}74,37 \\
(s d=2,28)\end{array}$ & $\begin{array}{c}78,59 \\
(s d=1,38)\end{array}$ \\
\hline Moyenne Communauté française de Belgique & $\begin{array}{c}74,40 \\
(s d=2,16)\end{array}$ & $\begin{array}{c}73,03 \\
(s d=1,46)\end{array}$ & $\begin{array}{c}77,37 \\
(s d=1,44)\end{array}$ \\
\hline
\end{tabular}


L'analyse du tableau 10 montre des différences de résultats selon le niveau des écoles sur l'indice socioéconomique (ISE), mais ne nous permet pas d'affirmer qu'il existe des différences significatives entre écoles Freinet et traditionnelles à niveau ISE similaire sur le plan des épreuves communes de référence.

\section{Discussion}

Nous avons réparti en deux groupes les classes des établissements scolaires Freinet et traditionnels de l'enseignement communal liégeois dont provenaient les élèves interrogés dans le cadre de cette recherche. Dans un premier groupe de classes provenant des établissements dont l'indice socioéconomique (ISE) vaut 1 ou 2 (deux écoles Freinet et deux traditionnelles) et dans un second groupe celles des établissements qui ont un indice ISE de 6 ou 7 (trois écoles Freinet et trois traditionnelles). Les élèves de ces écoles à qui nous avons fait passer le QES étaient inscrits dans le $3^{\mathrm{e}}$ cycle de l'enseignement primaire.

Notre première question de recherche portait sur les différences de résultats obtenus aux épreuves de référence chez les élèves, selon qu'ils faisaient partie d'une école Freinet ou d'une école dite traditionnelle de même catégorie ISE dans le réseau d'enseignement communal de la ville de Liège. Dans chacun des deux groupes, la comparaison des résultats aux épreuves communes de référence 2009-2011 ne montre pas de différences significatives entre les formes scolaires «Freinet » et «traditionnelle », que ce soit en français, en mathématiques ou en éveil. Ces résultats confortent les propos de LahanierReuter (2007) concernant le caractère comparable des connaissances acquises par les élèves de Freinet en mathématiques. De façon plus générale, en sciences, et en réponse aux interrogations quant à une éventuelle absence d'accès aux savoirs (Bonnéry, 2007; Lahire, 2008), les résultats de notre recherche ne montrent pas de moins-value en ce qui concerne les apprentissages en disciplines d'éveil au sein des écoles Freinet. En cela, ils font échos aux observations d'une autre chercheuse du collectif dirigé par Reuter, Cohen-Azria (2007), qui signale :

En confrontant différents axes d'analyse, l'école Freinet se distingue d'autres établissements étudiés par ses modes pédagogiques mis en place mais également par leurs effets. [...] En ce qui concerne l'enseignement des sciences et les apprentissages en sciences, cette étude permet de répondre que ces effets sont très bénéfiques. (Cohen-Azria, cité dans Reuter, 2007, p. 243)

Notre seconde question de recherche portait sur les différences de climat de classe en $5^{\mathrm{e}}$ et $6^{\mathrm{e}}$ au sein des écoles Freinet et traditionnelles de notre échantillon, à niveau ISE équivalent. Nous avons utilisé le Questionnaire sur l'environnement socioéducatif au primaire (QES) de Janosz et al. (2004), instrument québécois dont nous avons repris la partie «élèves ». Nous avons légèrement adapté cet instrument au contexte belge. Les questions du QES étaient réparties selon trois dimensions : I. Climat scolaire (21 items); II. Pratiques éducatives (38 items); III. Problématiques perçues et vécues dans l'école (56 items). Quelques questions d'informations générales (3 items) et liées à la motivation scolaire auto-révélée ( 5 items) figuraient également en fin de questionnaire. L'analyse des résultats montre des différences significatives en faveur de la forme scolaire «Freinet» pour toutes les variables des trois dimensions sauf pour les problèmes perçus de violence de gravité mineure, variable pour laquelle nous ne pouvons pas affirmer une différence significative. 
Nous avons aussi mesuré la force de ces différences concernant l'environnement socioéducatif. La dimension «I. Climat scolaire » comporte la variable dont la taille de l'effet est la plus forte en faveur des écoles Freinet, il s'agit de "La participation des élèves à la vie de l'école ». On observe aussi des tailles d'effet assez marquées dans cette première dimension pour les variables : "Climat relationnel entre les élèves et les enseignants »; "Climat de justice» et "Climat d'appartenance ». Ces résultats confortent les observations de Carra et Pagoni (2007) évoquées dans la section 4.1 ci-devant. Elles mettaient en évidence des valeurs ajoutées de la pédagogie Freinet en matière d'environnement socioéducatif et les expliquaient par la plus grande participation des élèves aux processus de régulations de la vie scolaire et notaient, entre autres, un sentiment de justice particulièrement fort dans l'école Freinet étudiée durant cinq ans.

Bien que des différences existent en faveur des écoles Freinet, les tailles d'effet sont moins marquées pour "Climat relationnel entre les élèves » et "Climat de sécuritéchez les élèves». Concernant la dimension "II. Pratiques éducatives de l'école", les différences en faveur de la forme scolaire Freinet sont assez marquées pour les variables "Pratiques pédagogiques » et la "Gestion des comportements en classe ». Les différences sont moins marquées pour les variables «Implantation et clarté des règles ", le "Temps consacré à l'enseignement » et le "Soutien aux élèves en difficulté ». Pour la dimension "III. Problématiques perçues et vécues dans l'école ", les différences sont assez marquées pour les "Comportements d'inadaptation scolaire perçus" et les "Problèmes de violence perçus de gravité majeure ", donc moins prégnants dans la forme scolaire Freinet. Des différences assez marquées en faveur de Freinet se manifestent aussi en matière de "Sécurité des lieux de l'école ». Il existe aussi des différences moins marquées en faveur de la forme scolaire Freinet en ce qui concerne les "Comportements d'inadaptation scolaire rapportés par les élèves", les "Problèmes de violence subis de gravité mineure et de gravité majeure ». Dans ce registre, l'étude menée par l'Observatoire international de la violence (Debarbieux, 2011) montre que la majorité des élèves vivent plutôt heureux à l'école et que les problèmes se concentrent sur une minorité de l'ordre de $10 \%$ soumis à un harcèlement qui peut s'avérer sévère pour la moitié d'entre eux (5 à 6 \%). Debarbieux (2012, p. 1) relate à ce sujet : « ... pour ces victimes, l'école peut être vécue comme un cauchemar. On rappellera qu'entre 20 et $25 \%$ des élèves absentéistes chroniques ne vont plus à l'école par peur de ce harcèlement (Blaya, 2010). ». Dès lors, nos constats en matière de valeur ajoutée par les établissements Freinet en lien avec la violence perçue et le sentiment de sécurité au sein de l'école sont loin d'être anecdotiques. Cette valeur ajoutée de l'école Freinet en matière de gestion des conflits et des comportements d'inadaptation scolaire renforce aussi d'autres constats sur la nécessité de travailler le cadre scolaire dans sa globalité dans une perspective systémique pour lutter efficacement contre le décrochage scolaire (Gilles, Tièche Christinat et Delevaux, 2012).

On retrouve aussi des différences moins marquées en faveur de Freinet pour la "Motivation scolaire auto-révélée ». Enfin, lorsqu'on agrège les variables au sein de chacune des trois dimensions, on observe des différences significatives assez marquées systématiquement en faveur de la forme scolaire Freinet. 


\section{Conclusion}

Dans le monde scolaire, la controverse sur les avantages de la pédagogie Freinet perdure depuis des décennies tant en ce qui concerne les valeurs ajoutées apportées en matière d'environnement socioéducatif que pour les compétences acquises au sein de cette forme d'enseignement lorsqu'elle est comparée aux écoles traditionnelles. La pédagogie Freinet est soumise à des remises en question telles que celles-ci :

- Puisque la pédagogie Freinet demande aux élèves de l'autonomie, l'agir autonome ne va-t-il pas masquer l'absence d'accès aux savoirs? (Bonnéry, 2007; Lahire, 2008);

- $\quad$ Les techniques Freinet ne donnent-elles pas la fausse impression de travailler en n'autorisant que certains à produire de la secondarisation? (Bakhtine, 1984; Bautier et Rochex, 2004).

Comme nous l'avons exposé plus haut, les recherches empiriques qui ont tenté de répondre à ces questions sont rares.

Notre étude a été réalisée dans le véritable laboratoire grandeur nature que constitue l'enseignement communal liégeois où plus de 1500 élèves, soit $14 \%$ de la population scolaire, sont inscrits dans des établissements Freinet. Nous avons été en mesure d'y comparer les formes scolaires Freinet et traditionnelles du point de vue de leurs environnements socioéducatifs auto-déclarés par les élèves et des résultats aux épreuves externes, et ce, dans des établissements similaires du point de vue du niveau socioéconomique moyen des quartiers d'habitation des élèves (indice ISE).

Notre étude montre qu'il n'y a pas de différence en ce qui concerne les quatre disciplines évaluées dans le cadre des épreuves externes de référence. En français, mathématiques, éveil-initiation scientifique et éveil-formation historique et géographique les résultats sont similaires. Les élèves de fin du primaire d'une forme scolaire ne performent pas mieux que ceux de l'autre.

L'analyse des résultats récoltés au QES montre que les élèves des écoles Freinet évaluent de façon plus positive leur environnement socioéducatif selon les trois dimensions mesurées par le questionnaire : climat scolaire, pratiques éducatives de l'école et problématiques perçues et vécues dans l'école. Les différences sont significatives et systématiquement en faveur des écoles Freinet.

Dans le contexte de l'enseignement communal liégeois, à niveau socioéconomique équivalent, les élèves des établissements Freinet obtiennent donc des résultats aux épreuves communes de fin du primaire similaires à ceux qu'obtiennent les élèves des écoles traditionnelles, mais s'en distinguent par des déclarations plus positives en ce qui concerne le climat scolaire et les pratiques éducatives. Les élèves des écoles Freinet déclarent également moins de problèmes scolaires au sens de Janosz et al. (1998). Autrement dit, en termes de valeur ajoutée de la pédagogie Freinet en fin du primaire, les résultats de notre recherche penchent pour une meilleure qualité d'environnement socioéducatif avec une équivalence des résultats en français, mathématiques et éveil. 


\section{Notes}

1 Le rapport de validation du QES-primaire peut être consulté en ligne : http://gres-umontreal.ca/download/Rapport validation OES-primaire.pdf

2 Ce domaine ne figure pas dans le CEB 2011.

3 Consulter ce lien : http://www.enseignement.be/index.php?page=26754\&navi=3376

$4 \quad$ Nous renvoyons au site web de l'enseignement communal liégeois pour plus d'informations sur les écoles fondamentales : http://www.ecl.be/ECL Site/2p ecoles/e 1 fond o/fo index.html

5 La version du questionnaire QES qui fut utilisée dans le cadre de notre recherche est accessible via ce lien: https://goo.gl/PE7kZb

6 Ce résultat avait été obtenu lors des analyses de variance « exploratoires » non reportées ici par violation d'hypothèses.

\section{Références}

Acker, V. (2006). Célestin Freinet : (1896-1966) - L'histoire d'un jeune intellectuel. Paris : L'Harmattan.

Bakhtine, M. M. (1984). Esthétique de la création verbale. Paris : Gallimard.

Bautier, É., Charlot, B. et Rochex, J.-Y. (2000). Entre apprentissage et métier d'élève : le rapport au savoir. Dans A. van Zanten (dir.), L'école, l'état des savoirs (p. 179-188). Paris : La Découverte.

Bautier, É. et Rochex, J.-Y. (1997). Apprendre : des malentendus qui font la différence. Dans J.-P. Terrail (dir.), La scolarisation de la France. Critique de l'état des lieux (p. 105-122). Paris : La Dispute.

Bautier, É. et Rochex, J.-Y. (2004). Activité conjointe ne signifie pas signification partagée. Raisons éducatives, (8), $199-220$.

Bertot, F. (2004). Éducation à la citoyenneté et débat : analyse comparative de deux pratiques pédagogiques. Spirale, (34), 5574. http://dx.doi.org/10.3406/spira.2004.1351

Blaya, C. (2010). Décrochages scolaires : l'école en difficulté. Bruxelles : De Boeck.

Bonnéry, S. (2007). Comprendre l'échec scolaire. Élèves en difficulté et dispositifs pédagogiques. Paris : La Dispute.

Carra, C. et Pagoni, M. (2007). Construction des normes et violences scolaires. Dans Y. Reuter (dir.), Une école Freinet - Fonctionnements et effets d'une pédagogie alternative en milieu populaire (p. 31-62). Paris : L'Harmattan.

CDA. (2009). Décret organisant un encadrement différencié au sein des établissements scolaires de la Communauté française afin d'assurer à chaque élève des chances égales d'émancipation sociale dans un environnement pédagogique de qualité. [D. 30-042009 M.B. 09-07-2009 - Modification : D. 08-07-10 (M.B. 16-08-10)]. Parlement de la Communauté française de Belgique, Centre de documentation administrative (CDA) de la Fédération Wallonie-Bruxelles.

Charlier, P. (2014, février). Le réseau d'écoles «Freinet » au sein de la ville de Liège. Communication présentée lors du Colloque "Freinet et l'école moderne aujourd'hui », Lausanne. Repéré à https://www.hepl.ch/cms/accueil/actualites-et-agenda/ archives-actualites/archives-2014/evenement-freinet/colloque/ateliers/atelier-4--le-reseau-decoles-fre.html

Cohen-Azria, C. (2007). Enseignement et apprentissages scientifiques. Dans Y. Reuter (dir.), Une école FreinetFonctionnements et effets d'une pédagogie alternative en milieu populaire (p. 217-244). Paris : L'Harmattan.

Debarbieux, E. (2011). Une enquête nationale de victimation et climat scolaire auprès d'élèves du cycle 3 des écoles élémentaires. À l'école des enfants heureux... enfin presque. Repéré à http://www.unicef.fr/userfiles/UNICEF FRANCE violences scolaires mars 2011.pdf

Debarbieux, E. (2012). Enquête de victimation et de climat scolaire auprès des personnels de l'éducation nationale en Seine-SaintDenis. Repéré à http://www.dsden93.ac-creteil.fr/spip/IMG/pdf/Conclusion_enquete_victimation-1.pdf

Debarbieux, E., Anton, N., Astor, R. A., Benbenishty, R., Bisson-Vaivre, C., Cohen, J., . . Vrand, R. (2012). Le « climat scolaire» : définition, effets et conditions d'amélioration. Repéré à http://www.cafepedagogique.net/lexpresso/Documents/docsjoints/climat-scolaire2012.pdf

De Bilde, J., Van Damme, J., Lamote, C. et De Fraine, B. (2013). Can alternative education increase children's early school engagement? A longitudinal study from kindergarten to third grade. School Effectiveness and School Improvement, 24(2), 212-233. http://dx.doi.org/10.1080/09243453.2012.749795

Fédération Wallonie-Bruxelles. (2012). Les indicateurs de l'enseignement (7éd.). Repéré à http://www.enseignement.be/download.php?do_id=9636 
Freinet, C. (1946a). L'école moderne française. Paris : Ophrys.

Freinet, C. (1946b). La coopération scolaire à l'École moderne. L'Éducateur, (18).

Freinet, C. (1969). Pour l'école du peuple : guide pratique pour l'organisation matérielle, technique et pédagogique de l'école populaire. Paris : Maspero.

Freinet, C. (1978). Les dits de Mathieu : une pédagogie moderne de bon sens. Paris : Delachaux et Niestlé.

Freinet, E. (1981). Naissance d'une pédagogie populaire. Paris : Maspero.

Gilles, J.-L., Tièche Christinat, C. et Delevaux, O. (2012). Origine, fondements et perspectives offertes par les alliances éducatives dans la lutte contre le décrochage scolaire. Dans J.-L. Gilles, P. Potvin et C. Tièche Christinat (dir.), Les alliances éducatives pour lutter contre le décrochage scolaire (p. 3-20). Berne : Peter Lang.

Go, H. L. (2006). Vers une reconstruction de la forme scolaire : l'institution du paysage à l'école Freinet de Vence. Carrefours de l'éducation, 22, 83-93. http://dx.doi.org/10.3917/cdle.022.0083

Goigoux, R. (2010). Une pédagogie éclectique au service des élèves qui ont le plus besoin de lécole. La nouvelle revue de l'adaptation et de la scolarisation, 52(4), 21-30. http://dx.doi.org/10.3917/nras.052.0021

Gottfredson, G. D. (1984). The effective school battery: user manual. Odessa, FL : Psychological Assessment Resources.

Janosz, M. (2000, novembre). The Montreal school environment project: The theoretical model and the validity of the School socioeducational environment questionnaire. Communication présentée à the Annual Meeting of the American Society of Criminology, San Francisco, CA.

Janosz, M., Bouthillier, C., Bowen, F., Chouinard, R. et Desbiens, N. (2007). Rapport de validation du Questionnaire sur l'environnement socioéducatif des écoles primaires (QES-primaire). Repéré à http://gres-umontreal.ca/download/Rapport_validation_QES-primaire.pdf

Janosz, M., Bowen, F., Chouinard, R. et Desbiens, N. (2004). Le Questionnaire d'évaluation de l'environnement socioéducatif pour écoles primaires. Montréal, QC : Université de Montréal.

Janosz, M., Georges, P. et Parent, S. (1998). L’environnement éducatif à l'école secondaire : un modèle théorique pour guider l'évaluation du milieu. Revue canadienne de psychoéducation, 27(2), 285-306.

Lahire, B. (2008). La raison scolaire. École et pratiques d'écriture, entre savoir et pouvoir. Rennes : Presses universitaires de Rennes.

Lahanier-Reuter, D. (2007). Enseignement et apprentissages mathématiques. Dans Y. Reuter (dir.), Une école Freinet - Fonctionnements et effets d'une pédagogie alternative en milieu populaire (p. 185-216). Paris : L'Harmattan.

Moos, R. H. et Trickett, E. J. (1974). The Classroom environment scale. Palo Alto, CA : Consulting psychologist press.

Pagoni, M. et Haeberli, P. (2006, juin). Conseils d'élèves et savoirs en éducation civique et morale : une étude comparative de deux établissements scolaires en France et en Suisse. Communication présentée au colloque international de l'AFEC "L'École, lieu de tensions et de médiations : quels effets sur les pratiques scolaires? Analyses et comparaisons internationales », Villeneuve-d'Ascq.

Peyronie, H. (2013). Le mouvement Freinet, du fondateur charismatique à l'intellectuel collectif. Caen : Presses universitaires de Caen.

Reuter, Y. (dir.) (2006). Effets d'un mode de travail pédagogique «Freinet» en REP. Rapport de recherche (2004-2006) remis à l'IUFM du Nord - Pas-de-Calais, Université Charles-de-Gaulle - Lille 3.

Reuter, Y. (2007). Une école Freinet, Fonctionnements et effets d'une pédagogie alternative en milieu populaire. Paris : LHarmattan.

Reuter, Y. et Carra, C. (2005). Analyser un mode de travail pédagogique « alternatif » : l'exemple d'un groupe scolaire travaillant en pédagogie « Freinet ». Revue française de pédagogie, (153), 39-53. http://dx.doi.org/10.3406/rfp.2005.3392

Saint-Luc, F. (2011). De la confrontation coopérative interculturelle à l'autoformation coopérative : le cas des formateurs d'enseignants au sein du mouvement international de l'École Moderne (Thèse de doctorat, Université d'Aix-Marseille). Repéré à https:// www.icem-pedagogie-freinet.org/sites/default/files/these saint luc florence.pdf

\section{Pour citer cet article}

Gilles, J.-L., Charlier, P., Pfeuti, S. et Prosperi, O. (2018). Appréciations par les élèves de leur environnement socioéducatif et résultats aux épreuves externes communes en fin d'enseignement primaire : comparaison des établissements Freinet et traditionnels de l'enseignement communal liégeois. Formation et profession, 26(3) 20-42.

http://dx.doi.org/10.18162/fp.2018.457 